Surgical Pearls I AAFPRS ANNUAL FALL MEETING FEATURED ARTICLE

\title{
Transcutaneous Skin-Muscle Flap Suspension Lower-Eyelid Blepharoplasty
}

Scott Shadfar, MD; Stephen W. Perkins, MD

The transcutaneous lower-eyelid blepharoplasty is a commonly performed surgical technique and an important tool in the armamentarium of facial plastic surgeons. The facets of lower-eyelid blepharoplasty begin with proper patient selection and rely on several technical subtleties that are crucial to achieve an excellent cos- metic and functional result while avoiding potential complications. The senior author (S.W.P) has been using the transcutaneous approach with orbicularis oculi muscle suspension for 30 years to address redundant or excessive skin and muscle while gaining wide exposure to treat pseudoherniation of the orbital fat compartments.

\section{Figure 1. The Transcutaneous Lower-Eyelid Blepharoplasty Technique}

A Subcilary incision

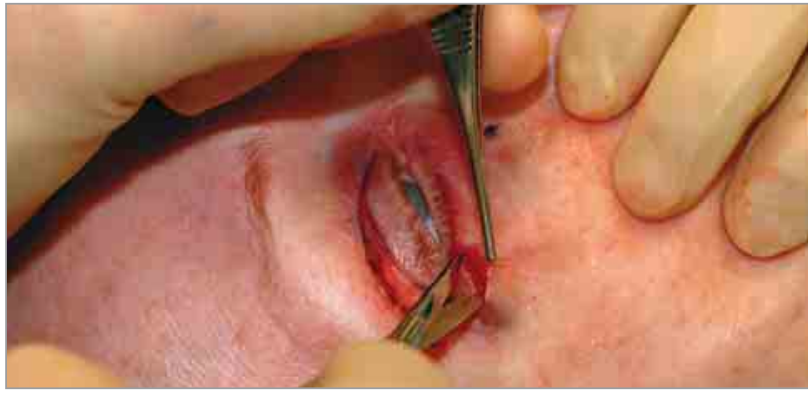

C Cauterize herniated fat

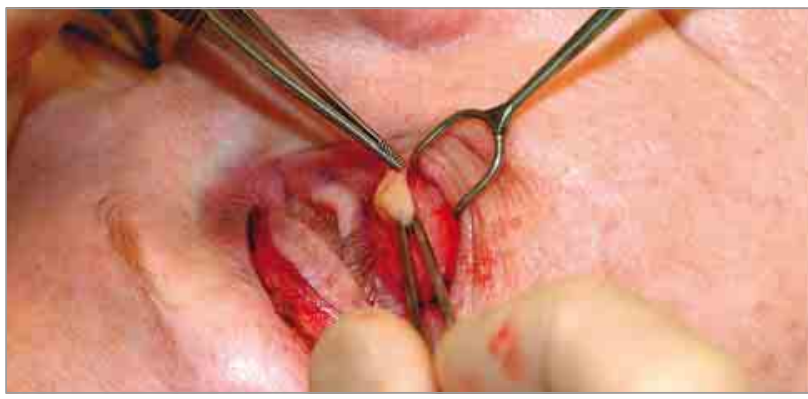

E Orbicularis oculi muscle suspension

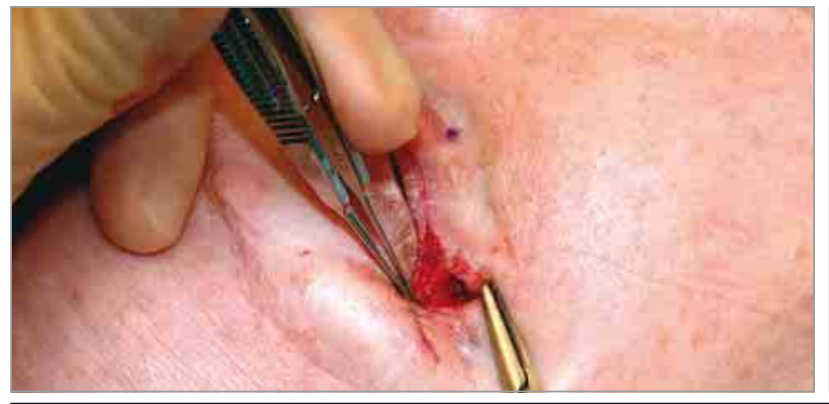

A and $B, A$ subciliary incision is made, the orbital septum is identified at the lateral aspect, and blunt scissors are used to elevate the skin-muscle flap off of the orbital septum bluntly (spreading) in an avascular plane. C, Herniated fat is gently grasped, and bipolar cautery is used to cauterize the base of each fat pad prior to excision. D, While the assistant holds digital pressure, an inferiorly
B Elevate skin-muscle flap

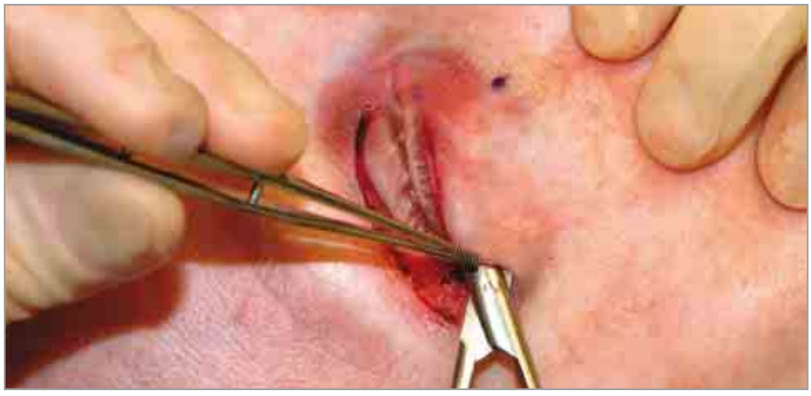

D Lateral canthus skin tailoring

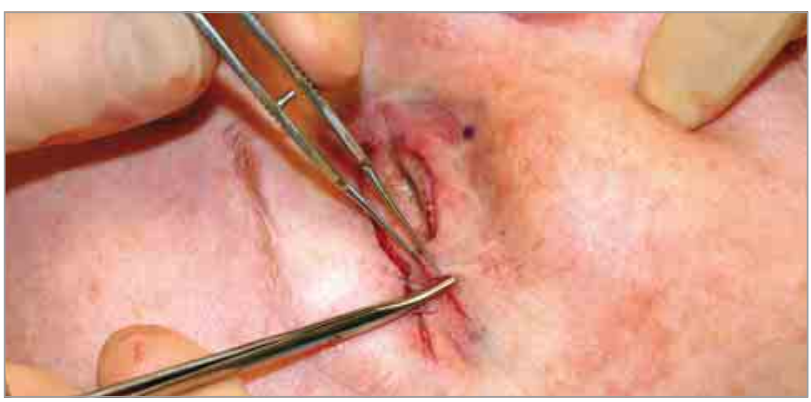

F Suspended lower eyelid

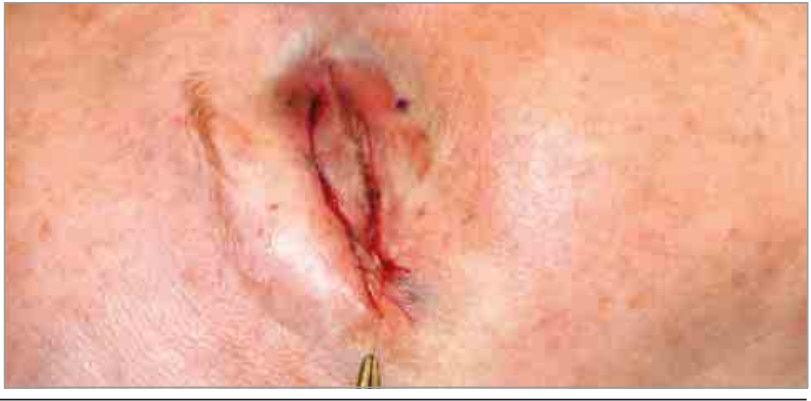

directed incremental cut is made in the skin at the lateral canthus, and the overlapping skin is conservatively excised. $E$, The periosteum of the lateral orbital rim at the level of tubercle is grasped using a 5-0 Monocryl suture (Ethicon), and then the suture is passed through the orbicularis oculi muscle on the skin-muscle flap. F, The lower eyelid is suspended. 
A Preoperative photograph

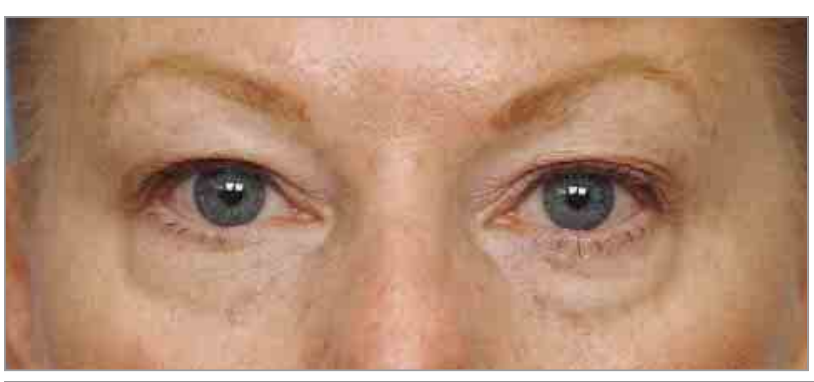

Surgical Technique

The transcutaneous skin-muscle flap is the most commonly used technique by the authors. The indications for this technique include true vertical excess of lower eyelid skin, orbicularis oculi muscle hypertrophy, and pseudoherniation of orbital fat., ${ }^{1,2}$

The procedure begins with a skin incision placed 2 to $3 \mathrm{~mm}$ inferior to the ciliary margin extending medially from the lower punctum to a position 6 to $8 \mathrm{~mm}$ lateral to the lateral canthus within a natural skin crease. Lateral extension of the incision to this position minimizes rounding of the canthal angle, provides adequate visualization, and allows for orbicularis oculi muscle suspension.

A pocket is created in the lateral aspect of the incision to identify the glistening white of the orbital septum (Figure 1). Blunt scissors are positioned posterior to the muscle at the lateral aspect of the incision; the skin-muscle flap is elevated off the orbital septum bluntly (spreading) in an

Video at jamafacialplasticsurgery.com avascular plane (Figure 1). The subciliary incision also involves pre-elevating the pretarsal skin flap to ensure the preservation of the pretarsal portion of the orbicularis oculi muscle. Using a bevel cut, the incision is completed, resulting in a stair-step configuration. This minimizes the risk of postoperative lower eyelid malposition.

Small openings overlaying the lateral, central, and medial compartments are made in the exposed orbital septum to obtain access to orbital fat. Gentle ballottement of the globe results in herniation of orbital fat through the openings created in the orbital septum. Herniated fat is gently grasped, and bipolar cautery is used at the base of each fat pad prior to excision (Figure 1). Careful hemostasis is obtained due to the risk of bleeding fat retracting posterior to the septum leading to postoperative hematoma formation. We take a conservative approach to fat resection to avoid the creation of a hollow appearance postoperatively. The remaining fat volume is assessed by gentle palpation of the globe following resection.

Preoperatively, the patients should be examined for physical findings of a deep nasojugal groove or tear trough deformity. In these cases, fat transposition of the nasal (medial) fat pocket, and occasionally central fat pocket, is performed. The stalk of the visualized flap is positioned inferiorly beneath the orbicularis oculi and sutured to the periosteum using a braided absorbable suture. The aim is to efface the depression in the nasojugal area while preserving or repurposing the orbital fat pocket.

When ready to redrape the skin-muscle flap, an assistant applies single-finger pressure at the inferomedial portion of the
B Postoperative blepharoplasty photograph at 6 mo

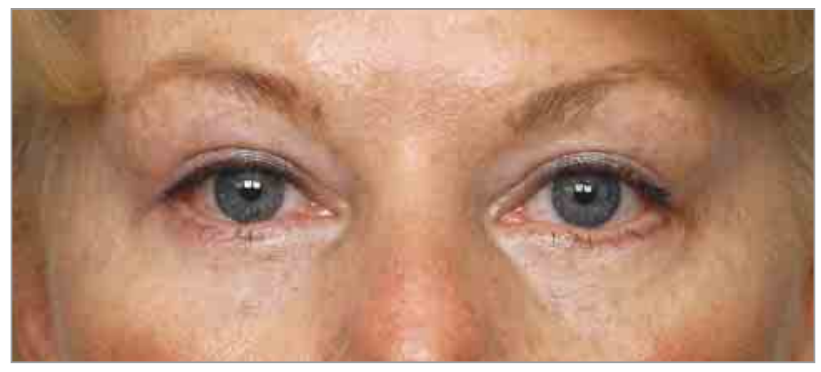

melolabial mound to create the same maximal stretch effect, similar to the awake patient opening his or her mouth and looking up. This maneuver helps prevent the surgeon from overresection of skin and muscle. An inferiorly directed incremental cut is made in the skin at the lateral canthus, and the overlapping skin is conservatively excised (Figure 1). If orbicularis oculi muscle hypertrophy is evident, a 1- to 2-mm strip of muscle is resected to prevent overlapping of muscle and ridge formation with closure of the subciliary incision. $^{3}$

The orbicularis oculi muscle is then suspended to the periosteum of the lateral orbital rim using a 5-0 Monocryl suture (Ethicon) in a buried fashion (Figure 1). Following muscle suspension, the lateral aspect of the incision is closed with 7-0 blue polypropylene suture (Ethicon) in a simple, interrupted fashion. The remainder of the subciliary incision is closed with 6-0 fast absorbing gut suture (Ethicon) in a simple running fashion. The sutures are removed 1 week postoperatively, and patients are free to use makeup.

Postoperative swelling may interfere with normal tear production and flow leading to temporary epiphora and dry eyes. It is imperative to instruct the patient about the importance of artificial tear use throughout the day and lubricating ophthalmic ointment placed onto the conjunctiva in the evenings.

This procedure can be viewed in full in the Video.

\section{Discussion}

The transcutaneous approach to lower-eyelid blepharoplasty has been met with some resistance due to concern for the development of retraction, lateral canthal rounding, scleral show, and ectropion. We and others ${ }^{1,4-6}$ have described techniques and experiences using the skin-muscle flap procedure with a low incidence of these complications. The pearls to achieve a well-supported and safe lower eyelid following lower-eyelid blepharoplasty (Figure 2) include meticulous hemostasis using bipolar cautery, conservative trimming and excision of redundant muscle and skin, as well as lateral suspension of the orbicularis oculi muscle. ${ }^{6}$

\section{Conclusions}

Transcutaneous lower-eyelid blepharoplasty can be an effective technique for lower eyelid rejuvenation. Important considerations for surgery include proper patient selection, careful review of the patient's ocular health history, and a thorough examination focusing on eyelid laxity. The skin-muscle flap method, when performed as described herein, carries minimal risk and yields natural and predictable results. 
ARTICLE INFORMATION

Author Affiliations: Meridian Plastic Surgeons, Indianapolis, Indiana (Shadfar, Perkins);

Department of Otolaryngology/Head and Neck Surgery, Indiana University School of Medicine, Indianapolis (Shadfar, Perkins).

Corresponding Author: Stephen W. Perkins, MD, 170 W 106th St, Indianapolis, IN 46240 (sperkski @gmail.com).

Published Online: October 3, 2015.

doi:10.1001/jamafacial.2015.1332.

Conflict of Interest Disclosures: None reported.

Previous Presentation: This article was presented at the American Academy of Facial Plastic and
Reconstructive Surgery Fall Meeting; October 3 , 2015; Dallas, Texas.

Additional Contributions: We thank Nancy Rothrock, MFA, for assistance with the article videography and photographs. Ms Rothrock was not compensated beyond her duties as an employee of Meridian Plastic Surgeons.

\section{REFERENCES}

1. Perkins SW, Holden P. Massry GG, Murphy MR, Azizzadaeh B, eds. Master Techniques in Blepharoplasty and Periorbital Rejuvenation. New York, NY: Springer Science + Business Media, LLC; 2011.

2. Perkins SW, Prischmann J. The art of blepharoplasty. Facial Plast Surg. 2011;27(1):58-66.
3. Bernardi C, Dura S, Amata PL. Treatment of orbicularis oculi muscle hypertrophy in lower lid blepharoplasty. Aesthetic Plast Surg. 1998;22(5): 349-351.

4. Honrado CP, Pastorek NJ. Long-term results of lower-lid suspension blepharoplasty. Arch Facial Plast Surg. 2004;6(3):150-154.

5. Prischmann J, Sufyan A, Ting JY, Ruffin C, Perkins SW. Dry eye symptoms and chemosis following blepharoplasty. JAMA Facial Plast Surg. 2013;15(1): 39-46.

6. Batniji RK, Perkins SW. Upper and midfacial rejuvenation in the non-Caucasian face. Facial Plast Surg Clin North Am. 2010;18(1):19-33. 